\title{
Isolation of an antimicrobial-resistant, biofilm-forming, Klebsiella grimontii isolate from a reusable water bottle
}

\author{
Alasdair T. M. Hubbard ${ }^{1}$ (D) | Enas Newire ${ }^{2}$ (D) | João Botelho ${ }^{3,4}$ (i) | Jesús Reiné ${ }^{5}$ (i) | \\ Elli Wright $^{1}$ | Emma A Murphy ${ }^{1}$ | William Hutton ${ }^{1}$ | Adam P. Roberts $^{1}$ (D)
}

${ }^{1}$ Department of Tropical Disease Biology, Liverpool School of Tropical Medicine, Liverpool, UK

${ }^{2}$ School of Pharmacy, College of Science, University of Lincoln, Lincoln, UK

${ }^{3}$ Antibiotic Resistance Evolution Group, Max-Planck-Institute for Evolutionary

Biology, Plön, Germany

${ }^{4}$ Department of Evolutionary Ecology and Genetics, Zoological Institute, ChristianAlbrechts-Universität zu Kiel, Kiel, Germany

${ }^{5}$ Department of Clinical Sciences, Liverpool School of Tropical Medicine, Liverpool, UK

Correspondence

Adam P. Roberts, Department of Tropical

Disease Biology, Liverpool School of Tropical

Medicine, Liverpool L3 5QA, UK.

Email: Adam.Roberts@lstmed.ac.uk

\begin{abstract}
A reusable water bottle was swabbed as part of the citizen science project "Swab and Send," and a Klebsiella grimontii isolate was recovered on chromogenic agar and designated SS141. Whole-genome sequencing of SS141 showed it has the potential to be a human pathogen as it contains the biosynthetic gene cluster for the potent cytotoxin, kleboxymycin, and genes for other virulence factors. The genome also contains the antibiotic-resistant genes, $b / a_{\mathrm{OxY}-6-4}$, and a variant of fosA, which is likely to explain the observed resistance to ampicillin, amoxicillin, and fosfomycin. We have also shown that SS141 forms biofilms on both polystyrene and polypropylene surfaces, providing a reasonable explanation for its ability to colonize a reusable water bottle. With the increasing use of reusable water bottles as an alternative to disposables and a strong forecast for growth in this industry over the next decade, this study highlights the need for cleanliness comparable to other reusable culinary items.
\end{abstract}

\section{KEYWORDS}

antibiotic resistance, biofilm, environmental, Kleboxymycin, Klebsiella grimontii, water bottle

\section{1 | INTRODUCTION}

As the global antimicrobial resistance (AMR) crisis continues and exacerbates, the scientific community are engaged in not only trying to discover novel antimicrobials to treat antimicrobial-resistant infections (Choi, Hwang, \& Lee, 2014; Liu, Yang, et al., 2018) but also understand the drivers of resistance and potential reservoirs of resistant pathogens (Kotsanas et al., 2013; Li et al., 2018; Lowe et al., 2012).

Enterobacteriaceae are of increasing concern, so much so that some have been deemed "critical" on the WHO global priority list of antimicrobial-resistant pathogens. The pathogen Klebsiella grimontii is of particular importance as, although it can be part of the normal microflora of the gastrointestinal tract, it has been found to be an etiological agent of antimicrobial-associated hemorrhagic colitis (Passet
\& Brisse, 2018). Until recently, K. grimontii was thought to be a phylogroup of Klebsiella oxytoca; however, it was found to be a distinct pathogenic species of Klebsiella and renamed K. grimontii and characterized in part by the presence of the chromosomally located $\beta$-lactamase gene, blaOXY-6 (Liu, Feng, et al., 2018; Passet \& Brisse, 2018).

Swab and Send (LSTM, 2019) is an antibiotic discovery, citizen science project in which members of the public swab an environment of their choice and return the swab to our laboratory. The bacteria from these swabs are isolated and, using an in-house assay, the antibiotic-producing potential of the isolates is determined against indicator strains, including Micrococcus luteus, Escherichia coli, Candida auris, Candida albicans, and methicillin-resistant Staphylococcus aureus. As part of the Swab and Send project, a swab was taken from a reusable water bottle from the offices of the Telegraph Newspaper (Ough, 2018). When this swab was plated out on chromogenic agar, there

This is an open access article under the terms of the Creative Commons Attribution License, which permits use, distribution and reproduction in any medium, provided the original work is properly cited.

(c) 2020 The Authors. MicrobiologyOpen published by John Wiley \& Sons Ltd. 
was an abundance of growth of presumptive Enterobacteriaceae. Pure culture and sequencing showed it was likely to be an antibiotic-resistant, pathogenic strain of $K$. grimontii, which is able to form biofilms readily on abiotic surfaces.

\section{2 | MATERIALS AND METHODS}

\section{1 | Media and antimicrobials}

Ampicillin (AMP), amoxicillin ( $A M X)$, amoxicillin-clavulanic acid (AMC), ceftriaxone (CEF), olaquindox (OLA), and fosfomycin (FOS) were prepared in molecular grade water; ciprofloxacin (CIP) was prepared in $0.1 \mathrm{~N}$ hydrochloric acid solution; tetracycline was prepared in 50\% ethanol (all Sigma); and chloramphenicol (CHL; Sigma) was prepared in ethanol (VWR) all to a stock concentration of $1 \mathrm{mg} / \mathrm{ml}$.

Growth of SS141 from $-80^{\circ} \mathrm{C}$ stocks was on Lysogeny broth (Lennox) agar at $37^{\circ} \mathrm{C}$ for $18 \mathrm{hr}$ and subcultured into liquid culture using either Lysogeny broth (Lennox) or cation-adjusted Mueller Hinton Broth (all Sigma) at $37^{\circ} \mathrm{C}$ for $18 \mathrm{hr}$ at $200 \mathrm{rpm}$, unless stated.

\section{2 | Isolation of SS141}

A single sample was taken from the interior of the rim of a reusable water bottle using a Transwab ${ }^{\circledR}$ Amies Charcoal Swab (MWE Medical Wire $\&$ Equipment) and kept at ambient temperature. The swab was used to directly inoculate $\mathrm{CHROMagar}{ }^{\mathrm{TM}}$ Orientation (CHROMagar) chromogenic agar plate and incubated at $37^{\circ} \mathrm{C}$ for $18 \mathrm{hr}$. A single colony that was metallic green/blue in appearance was chosen and pure-cultured onto $\mathrm{CHROMagar}{ }^{\mathrm{TM}}$ Orientation agar. The pure isolate was then kept in stocks at $-80^{\circ} \mathrm{C}$ in $40 \%$ glycerol (Sigma, UK).

\subsection{Sequencing and bioinformatics}

Short read sequencing of isolate SS141 and subsequent read trimming were performed by MicrobesNG (MicrobesNG) using the Illumina MiSeq platform. Long read sequencing was performed using the Oxford Nanopore Technologies MinION, using the SQKLSK109 ligation and SQK-RBK103 barcoding kit, on an R9.4.1 flowcell, and all reads were basecalled via MinKNOW. Sequenced reads were demultiplexed, and the adapters were trimmed using Porechop (version 0.2.4) and filtered for a quality score of 30 using Filtlong (version 0.2.0). The whole-genome sequence of SS141 was assembled using both long and short reads with Unicycler via Galaxy (version 0.4.6.0; Wick, Judd, Gorrie, \& Holt, 2017), the quality statistics of each assembly were determined using QUAST (version 4.6.3; Mikheenko, Prjibelski, Saveliev, Antipov, \& Gurevich, 2018), and then, subsequently assembled contigs were annotated using Prokka (version 1.14.0; Seemann, 2014). Finally, the assembly graph was visualized using Bandage (version 0.8.1; Wick, Schultz, Zobel, \& Holt, 2015).
Using the assembled contigs, it was initially determined whether the isolate was pathogenic using PathogenFinder (version 1.1; Cosentino, Voldby Larsen, Moller Aarestrup, \& Lund, 2013) and compared with other strains of K. grimontii, K. oxytoca, and Klebsiella pneumoniae by calculating the average nucleotide identity (ANI) using OrthoANI (version 0.93.1; Lee, Ouk Kim, Park, \& Chun, 2016). Acquired antimicrobial resistance genes located in the whole genome sequence or plasmids were searched for using ResFinder (version 3.2; Zankari et al., 2012) with an identity threshold of $60 \%$ and a minimum coverage of $60 \%$, and metal resistance genes were identified using the BacMet database (version 2.0; Pal, BengtssonPalme, Rensing, Kristiansson, \& Larsson, 2014). Finally, biosynthetic gene clusters (BGC) in the whole genome sequence were searched for using antiSMASH (version 5.0; Blin et al., 2019) and the kleboxymycin biosynthetic gene cluster characterized using SnapGene software (version 3.3.4).

\subsection{Biofilm assays}

The 96-well plate biofilm assay was performed as described previously (Hubbard, Jafari, Feasey, Rohn, \& Roberts, 2019) in a 96-well plate made from either polystyrene $\left(\operatorname{Costar}^{\circledR}\right.$ round bottom, nontreated, sterile, microplates; Corning Life Sciences) or polypropylene (U-bottom, sterile 96 well microplate; Greiner Bio-One). Briefly, following incubation in Luria Bertani broth, each culture was diluted $1 / 1,000$ in M9 $(50 \%$ (v/v) M9 minimal salts (2x) (Gibco, Thermo Fisher Scientific), 0.4\% D-glucose, $4 \mathrm{mM}$ magnesium sulfate (both Sigma), and $0.05 \mathrm{mM}$ calcium chloride (Millipore) and incubated for $24 \mathrm{hr}$ at either room temperature or $37^{\circ} \mathrm{C}$, statically. Following incubation, each culture was washed four times in PBS (PBS, pH 7.2; Gibco, Thermo Fisher Scientific), stained with $0.1 \%$ crystal violet (Sigma) followed by washing four times in PBS, and the stained biofilm dissolved in 30\% acetic acid (Fisher Scientific). Finally, biofilm production was quantified at an optical density of $550 \mathrm{~nm}\left(\mathrm{OD}_{550}\right)$ using a microplate reader.

Single tube biofilm assays were performed in 15-ml centrifuge tubes made from either polystyrene or polypropylene (Falcon ${ }^{\circledR}$; Corning Life Sciences). Following incubation Luria Bertani broth, each culture was diluted 1/1,000 in $4 \mathrm{ml}$ of $\mathrm{M} 9$ and incubated statically at $37^{\circ} \mathrm{C}$ or room temperature for $24 \mathrm{hr}$ alongside a negative control of $4 \mathrm{ml} \mathrm{M9}$ only. After incubation, each tube was washed four times with $6 \mathrm{ml}$ of PBS and left to dry for 30-60 min and then stained with $5 \mathrm{ml} 0.1 \%$ crystal violet solution for $15 \mathrm{~min}$. The crystal violet stain was washed off with four washes of $6 \mathrm{ml} \mathrm{PBS}$ and left to dry for 60-120 min and photographed.

\section{5 | Minimum inhibitory concentrations}

Minimum inhibitory concentration (MIC) of AMP, AMX, AMC, CEF, CIP, OLA, CHL, and FOF to SS141 were carried out using cationadjusted Mueller Hinton Broth following the CLSI guidelines for 
FIGURE 1 Calculation of the percentage Average Nucleotide Identity (ANI) of K. grimontii SS141, K. grimontii WCHKG020121, K. grimontii 06D021, K. oxytoca 09-7231-1, K. oxytoca E718, K. oxytoca JKo3, K. pneumoniae KPL0.1, and K. pneumoniae KPLO.2. ${ }^{*}$ K. oxytoca E718 was submitted as Klebsiella michiganensis on GenBank. Figures under the isolate name represent the probability of being a human pathogen, as determined by PathogenFinder

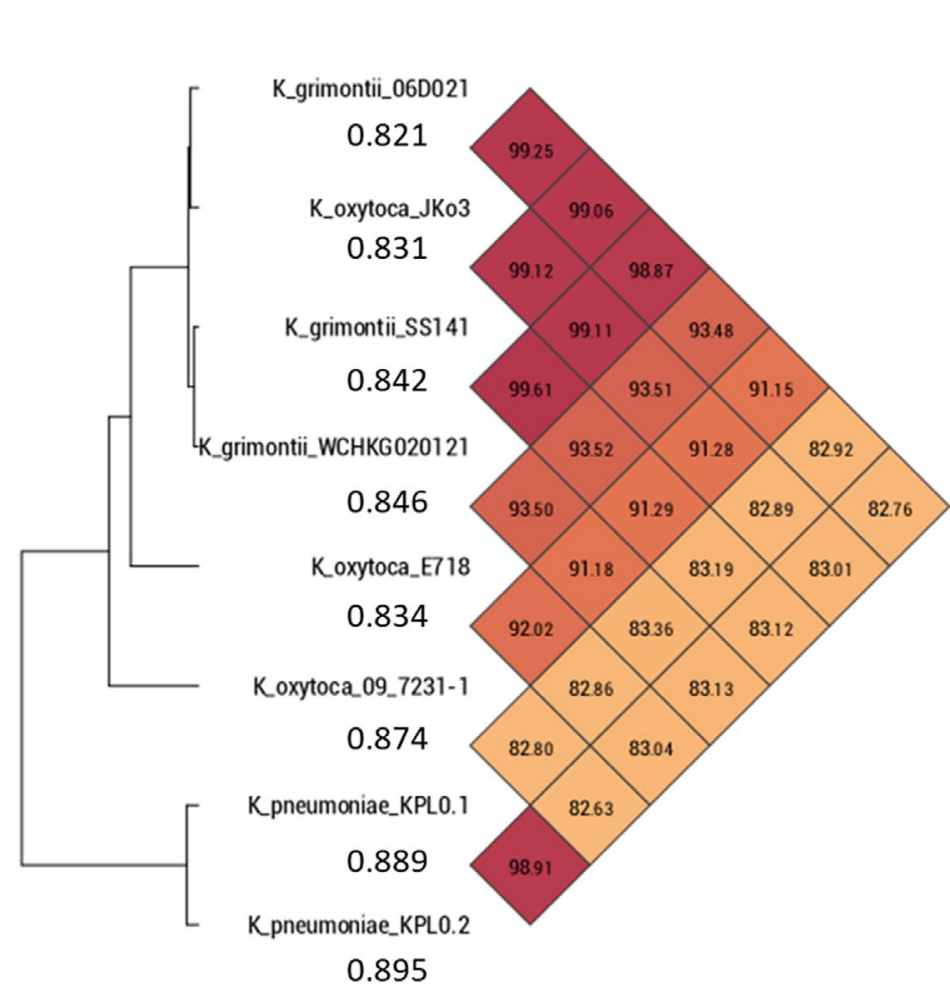

antimicrobial susceptibility testing using the broth microdilution methods (CLSI, 2018).

\section{RESULTS AND DISCUSSION}

The swab taken from a reusable water bottle was initially plated out on to the chromogenic CHROMagar ${ }^{\mathrm{TM}}$ Orientation agar routinely used for the identification of urinary tract pathogens (Samra, Heifetz, Talmor, Bain, \& Bahar, 1998), which identified a significant amount of presumptive Enterobacteriaceae present on the swab. Due to the metallic blue/green pigmentation and morphology of the colonies on the chromogenic agar, it was determined that these colonies were probable Citrobacter, Enterobacter, or Klebsiella, which are often pathogenic. We therefore subcultured until it was a pure isolate, denoted as isolate SS141.

Following a hybrid assembly of the whole genome sequencing data, the isolate was identified as $K$. grimontii. It contains two low copy number plasmids: IncFII(K) (101 kb in size and 4.11× depth) and IncFIA(HI) (134 kb in size and 1.98× depth) and a small extrachromosomal molecule, which was unable to be fully resolved and with extremely high copy number (between 1,348.61 and 1,357.92x depth). Antimicrobial resistance genes were not found to be present on any of these plasmids; however, putative heavy metal-resistant genes (silE, cusA, pcoR, and copA/B/C/D potentially conferring resistance to silver and copper) were found to be present on the IncFIA(HI1) plasmid and arsR, implicated in resistance to arsenic, was found on the chromosome of SS141. While K. grimontii can be asymptomatically carried by humans, it has also been associated with the cause of a number of infections including antibiotic-associated hemorrhagic colitis and bacteremia (Passet \& Brisse, 2018). Using PathogenFinder, we found SS141 is likely to be a human pathogen with a probability score of 0.842 . Subsequently, we compared the SS141 genome to a clinical strain isolated from a patient's sputum, K. grimontii WCHKG020121 (Liu, Feng, et al., 2018); the reference strain K. grimontii 06D021 (Passet \& Brisse, 2018); three published genomes of pathogenic strains of K. oxytoca: $K$. oxytoca 09-7231-1 isolated from a mouse tumor abscess (Darby et al., 2014), K. oxytoca E718*, a clinical isolate from Taiwan (Liao et al., 2012), and $K$. oxytoca $\mathrm{JKo3}$, a clinical isolate from a strain collection in Japan (Iwase, Ogura, Hayashi, \& Mizunoe, 2016); and two clinical isolates of K. pneumoniae (KPL0.1 and KPL0.2; Le Guern et al., 2018) using ANI (Figure 1). We found that SS141 was very closely related to the clinical isolate K. grimontii WCHKG020121 (99.61\%) and reference strain K. grimontii 06D021 (99.06\%), further confirming that SS141 is K. grimontii. SS141 was also $99.12 \%$ identical to K. oxytoca JKo3 suggesting that K. oxytoca JKo3 is likely to have been misidentified and is actually K. grimontii (Figure 1). All these strains had a

TAB LE 1 Minimum inhibitory concentrations ( $\mu \mathrm{g} / \mathrm{ml}$ ) of ampicillin (AMP), amoxicillin (AMX), amoxicillin-clavulanic acid (AMC), ceftriaxone (CEF), ciprofloxacin (CIP), olaquindox (OLA), chloramphenicol (CHL), and fosfomycin (FOF) to the SS141 isolate

\begin{tabular}{lllllllll} 
& AMP & AMX & AMC & CEF & CIP & OLA & CHL & FOF \\
SS141 & 16 & 32 & 2 & $0.03125-0.0625$ & 0.0078125 & 16 & $2-4$ & 256 \\
\hline
\end{tabular}




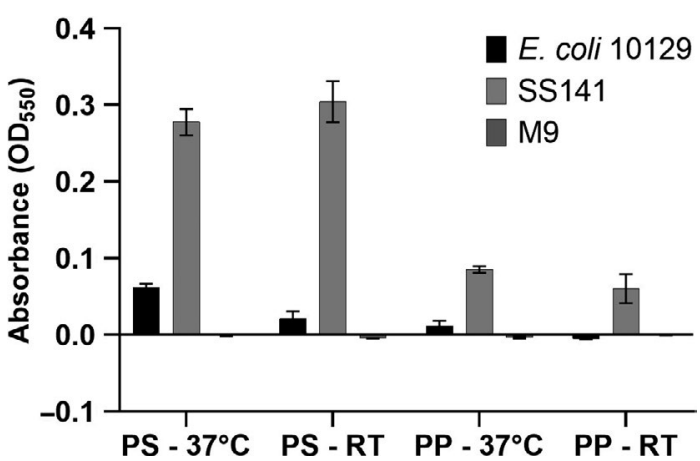

FIGURE 2 Biofilm formation (expressed as absorbance at $\mathrm{OD}_{550}$ of stained biofilm) of the SS141 isolate and E. coli 10129 in M9 in polypropylene (PP) and polystyrene (PS) 96 well plates following incubation at either room temperature (RT) or $37^{\circ} \mathrm{C}$. Error bars represent the standard error of the mean

similar likelihood of pathogenicity as determined by PathogenFinder (Figure 1).

Four antimicrobial resistance genes were identified to be present on the SS141 chromosome using ResFinder, $b a_{\text {OxY-6-4, }}$, which confers resistance to $\beta$-lactam antibiotics, including amoxicillin and ticarcillin (Fevre et al., 2005), and is an indicator of K. grimontii (Liu, Feng, et al., 2018; Passet \& Brisse, 2018). Both oqxA and oqxB, which are involved in resistance to olaquindox (Hansen, Johannesen, Burmolle, Sorensen, \& Sorensen, 2004), an antimicrobial used as a growth promoter in animals (Barber, Braude, Hosking, \& Mitchell, 1979), and a variant of the fosfomycin-resistant gene fosA were all also identified and were all previously found to be present on the chromosome of several other K. grimontii isolates (Liu, Feng, et al., 2018). Resistance to the $\beta$-lactam antimicrobials AMP and AMX was confirmed (MIC of 16 and $32 \mu \mathrm{g} / \mathrm{ml}$, respectively); however, SS141 was still sensitive to both AMC $(2 \mu \mathrm{g} / \mathrm{ml})$ and CEF $(0.0625 \mu \mathrm{g} / \mathrm{ml})$ according to EUCAST clinical breakpoints (Table 1). Resistance to fosfomycin was also confirmed with a MIC of $256 \mu \mathrm{g} / \mathrm{ml}$, which is well over the clinical breakpoint of $>32 \mu \mathrm{g} / \mathrm{ml}$. Despite the identification of oqxAB genes in SS141 using ResFinder, the isolate was sensitive to OLA (16 $\mu \mathrm{g} /$ $\mathrm{ml}$ ) according to the previously determined breakpoint of $>64 \mu \mathrm{g} /$ ml (Hansen et al., 2004; Liu, Wu, et al., 2018; Table 1). While oqxB2 was confirmed to be present in the genome following annotation using Prokka, we noticed that the oqxA gene identified by ResFinder was annotated by Prokka as bepF gene, which encodes for the protein BepF, part of the RND-type efflux pump BepFG, and has been shown to be involved in drug resistance to some degree in Brucella suis (Martin et al., 2009). Therefore, lack of confirmation of the presence of oqxA in the genome would explain the lack of resistance seen to olaquindox in the phenotypic assays. The oqxAB genes have also been reported to be present in the K. grimontii WCHKG020121 genome (Liu, Feng, et al., 2018), which align with 100\% identity to the oqxAB genes identified by ResFinder in SS141. This highlights an important consideration when assessing antibiotic resistance using genomic data and antibiotic-resistant gene databases as inaccurate annotations may result in antibiotic resistance being incorrectly attributed to an isolate.

The SS141 isolate genome contains treC, which has previously been shown to be involved biofilm formation in Klebsiella pneumoniae and is particularly important during the colonization of the (a)

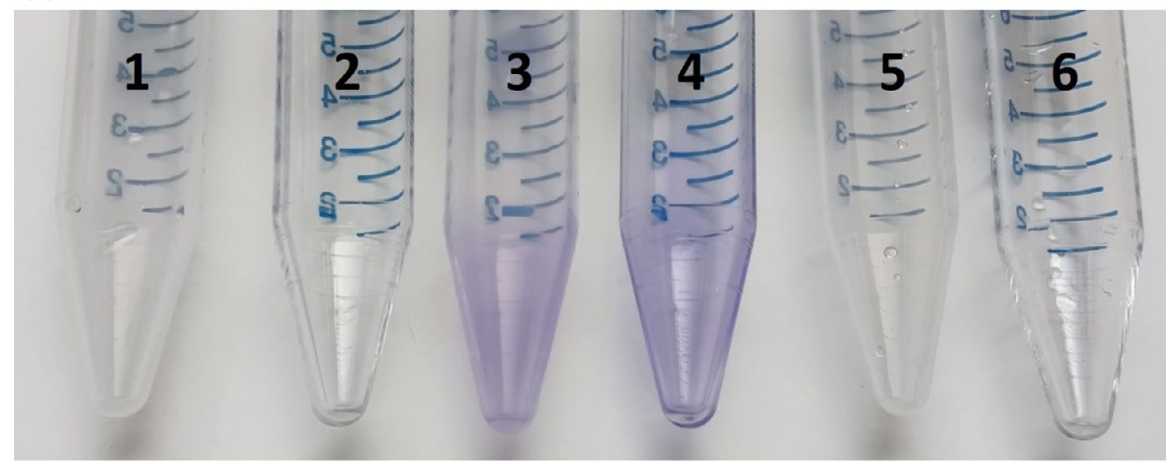

(b)

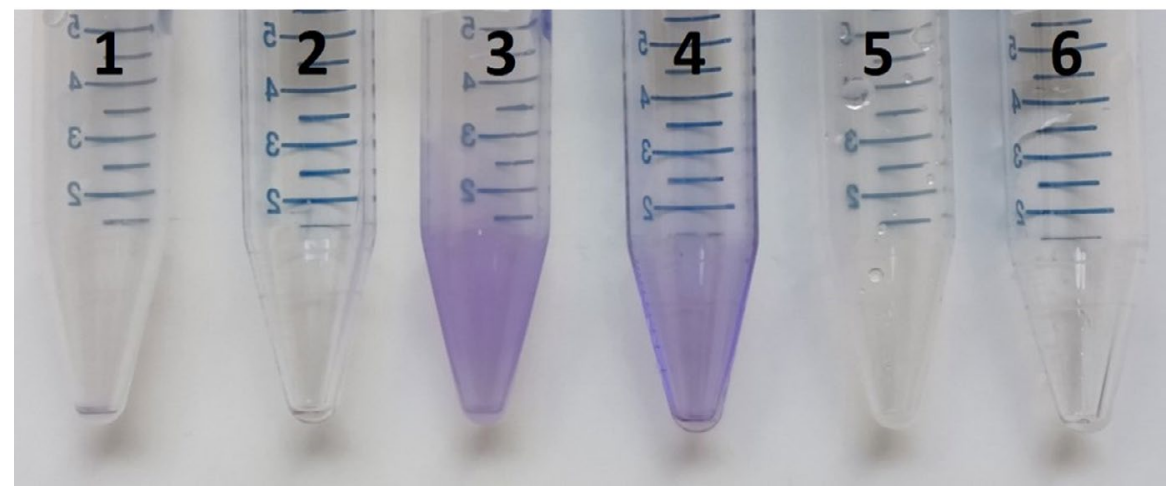

FIGURE 3 Representative photographs of biofilm formation of (1) E. coli 10129 in polypropylene, (2) E. coli 10129 in polystyrene, (3) SS141 in polypropylene, (4) SS141 in polystyrene, (5) M9 only in polypropylene, and (6) M9 only in polystyrene incubated at (a) $37^{\circ} \mathrm{C}$ and (b) room temperature for $24 \mathrm{hr}$. Repeat 2 and 3 of this experiment are provided in Figures $\mathrm{A} 1$ and $\mathrm{A} 2$ 
gastrointestinal tract (Wu, Lin, Hsieh, Yang, \& Wang, 2011). We have confirmed that SS141 is a biofilm former, producing a visible and significant biofilm during growth in the minimal media M9 at $37^{\circ} \mathrm{C}$ (Ordinary one-way ANOVA, uncorrected Fisher's LSD $p$ value $=<.0001$ ) and room temperature (Ordinary one-way ANOVA, uncorrected Fisher's LSD $p$-value $=<.0001$ ) compared with Escherichia coli 10129, a known biofilm producer (Figures 2 and 3a,; Hubbard et al., 2019). As biofilm formation on a polystyrene 96 -well plate and centrifuge tubes do not represent the same plastic commonly used in reuseable water bottles, we also tested the biofilm production potential of SS141 in centrifuge tubes and 96-well plates made from polypropylene, a significant component of reuseable water bottles. We found that SS141 also produces significant and visible biofilms on polypropylene at both $37^{\circ} \mathrm{C}$ (Ordinary one-way ANOVA, uncorrected Fisher's LSD $p$-value $=<.0001$ ) and room temperature (Ordinary oneway ANOVA, uncorrected Fisher's LSD $p$-value $=.0056$; Figures 2 and 3a,) compared with E. coli 10129. Although the biofilm production on polypropylene was significantly less than polystyrene at the same temperature $\left(37^{\circ} \mathrm{C}\right.$; Ordinary one-way ANOVA, uncorrected Fisher's LSD $p$-value $=<.0001$, room temperature; Ordinary oneway ANOVA, uncorrected Fisher's LSD $p$-value $=<.0001$ ), this would provide a good explanation for isolation of the Klebsiella from the swab and indicates that SS141 has the potential to persist in the environment, in this case on a reusable water bottle. While this study is limited to examining the biofilm production of SS141 on polystyrene and polypropylene surfaces, the ability of bacteria isolated from the environment to form biofilms on a range of plastic surfaces could be investigated, as well as the minimum inoculum size required to form biofilms on plastic surfaces. Finally, the BGC for the potent cytotoxin kleboxymycin was identified using antiSMASH and found to be present within the SS141 genome with $99.53 \%$ homology with the previously characterized kleboxymycin BGC (accession number MF401554; Tse et al., 2017). This cytotoxin has been previously associated with the cause of antibiotic-associated hemorrhagic colitis (Tse et al., 2017), further suggesting that SS141 is a pathogenic strain of $K$. grimontii.

\section{4 | CONCLUSIONS}

We describe the first instance of isolation of a $K$. grimontii isolate from a reusable water bottle. The isolate was found to form biofilms on polypropylene and polystyrene and carried multiple resistance genes to both metals and antibiotics. It is also likely to be pathogenic as the biosynthetic gene cluster for kleboxymycin is present within the genome. There is a strong forecast in the global market for reusable water bottles over the next decade (Accuray-ResearchLLP, 2019), and this study highlights the aspect of cleanliness when it comes to repeated use of such household items. There is, as yet, no comprehensive scientific study of bacterial colonization of reusable water bottles, and perhaps this is needed to persuade manufacturers and users to promote and practice suitable washing regimens aimed at keeping bacterial load to a minimum.

\section{ACKNOWLEDGMENTS}

We would like to thank MWE, Medical Wire \& Equipment, for their continued support of the Swab and Send citizen science project.

\section{CONFLICT OF INTEREST}

None declared.

\section{AUTHOR CONTRIBUTION}

Alasdair Hubbard: Conceptualization; Formal analysis; Investigation; Writing-original draft; Writing-review \& editing. Enas Newire: Formal analysis; Investigation; Writing-review \& editing. João Botelho: Formal analysis; Investigation; Writing-review \& editing. Jesús Reiné: Formal analysis; Investigation; Writing-review \& editing. Elli Wright: Formal analysis; Investigation; Writing-review \& editing. Emma A. Murphy: Formal analysis; Investigation; Writing-review \& editing. William Hutton: Formal analysis; Investigation; Writing-review \& editing. Adam P. Roberts: Conceptualization; Formal analysis; Writingoriginal draft; Writing-review \& editing.

\section{ETHICS STATEMENT}

None required.

\section{DATA AVAILABILITY STATEMENT}

This Whole Genome Shotgun project for Klebsiella grimontii SS141 has been deposited at GenBank under the following accession numbers; complete: circular chromosome accession number CP044527; complete, circular IncFII(K) plasmid accession number CP044529; complete, circular IncFIA(HI1) plasmid accession number CP044528; the incomplete high copy number plasmid accession numbers CP044530 and CP044531.

\section{ORCID}

Alasdair T. M. Hubbard (iD https://orcid.org/0000-0001-6668-9179 Enas Newire iD https://orcid.org/0000-0002-4023-8107

João Botelho (iD https://orcid.org/0000-0002-2771-2345

Jesús Reiné (iD https://orcid.org/0000-0001-7462-2054

Adam P. Roberts (iD https://orcid.org/0000-0002-0760-3088

\section{REFERENCES}

Accuray Research LLP (2019). Global reusable water bottles market analysis \& trends - industry forecast to 2027. Retrieved from https://www. researchandmarkets.com/research/9m8t4z/global_reusable? $\mathrm{w}=5$. Research and Markets.

Barber, R. S., Braude, R., Hosking, Z. D., \& Mitchell, K. G. (1979). Olaquindox as performance-promoting feed additive for growing pigs. Animal Feed Science and Technology, 4, 117-123. https://doi. org/10.1016/0377-8401(79)90036-1

Blin, K., Shaw, S., Steinke, K., Villebro, R., Ziemert, N., Lee, S. Y., ... Weber, T. (2019). antiSMASH 5.0: Updates to the secondary metabolite genome mining pipeline. Nucleic Acids Research, 47(W1), W81-W87. https://doi.org/10.1093/nar/gkz310

Choi, H., Hwang, J. S., \& Lee, D. G. (2014). Identification of a novel antimicrobial peptide, scolopendin 1 , derived from centipede Scolopendra subspinipes mutilans and its antifungal mechanism. Insect Molecular Biology, 23(6), 788-799. https://doi.org/10.1111/imb. 12124 
Clinical and Laboratory Standards Institute (2018). Methods for dilution antimicrobial susceptibility tests for bacteria that grow aerobically. In 11th Ed. CLSI Standard M07. Wayne, PA: CLSI.

Cosentino, S., Voldby Larsen, M., Moller Aarestrup, F., \& Lund, O. (2013). PathogenFinder-distinguishing friend from foe using bacterial whole genome sequence data. PLoS ONE, 8(10), e77302. https://doi. org/10.1371/journal.pone.0077302

Darby, A., Lertpiriyapong, K., Sarkar, U., Seneviratne, U., Park, D. S., Gamazon, E. R., ... Fox, J. G. (2014). Cytotoxic and pathogenic properties of Klebsiella oxytoca isolated from laboratory animals. PLoS ONE, 9(7), e100542. https://doi.org/10.1371/journal.pone.0100542

Fevre, C., Jbel, M., Passet, V., Weill, F. X., Grimont, P. A., \& Brisse, S. (2005). Six groups of the OXY beta-Lactamase evolved over millions of years in Klebsiella oxytoca. Antimicrobial Agents and Chemotherapy, 49(8), 3453-3462. https://doi.org/10.1128/AAC.49.8.3453-3462.2005

Hansen, L. H., Johannesen, E., Burmolle, M., Sorensen, A. H., \& Sorensen, S. J. (2004). Plasmid-encoded multidrug efflux pump conferring resistance to olaquindox in Escherichia coli. Antimicrobial Agents and Chemotherapy, 48(9), 3332-3337. https://doi.org/10.1128/ AAC.48.9.3332-3337.2004

Hubbard, A. T. M., Jafari, N. V., Feasey, N., Rohn, J. L., \& Roberts, A. P. (2019). Effect of environment on the evolutionary trajectories and growth characteristics of antibiotic-resistant Escherichia coli mutants. Frontiers in Microbiology, 10, 2001. https://doi.org/10.3389/ fmicb.2019.02001

Iwase, T., Ogura, Y., Hayashi, T., \& Mizunoe, Y. (2016). Complete genome sequence of Klebsiella oxytoca Strain JKo3. Genome Announcement, 4(6), e00438-16. https://doi.org/10.1128/genomeA.01221-16

Kotsanas, D., Wijesooriya, W. R. P. L. I., Korman, T. M., Gillespie, E. E., Wright, L., Snook, K., ... Stuart, R. L. (2013). "Down the drain": Carbapenem-resistant bacteria in intensive care unit patients and handwashing sinks. Medical Journal of Australia, 198(5), 267-269. https://doi.org/10.5694/mja12.11757

Le Guern, R., Grandjean, T., Faure, K., Bauduin, M., Kipnis, E., \& Desseina, R. (2018). Draft genome sequences of two Carbapenemase-producing Klebsiella pneumoniae strains isolated from blood cultures. Microbiology Resource Announcements, 7(8), e01057-01018. https:// doi.org/10.1128/MRA.01057-18

Lee, I., Ouk Kim, Y., Park, S. C., \& Chun, J. (2016). OrthoANI: An improved algorithm and software for calculating average nucleotide identity. International Journal of Systematic and Evolutionary Microbiology, 66(2), 1100-1103. https://doi.org/10.1099/ijsem.0.000760

Li, L., Dechesne, A., He, Z., Madsen, J. S., Nesme, J., Sørensen, S. J., \& Smets, B. F. (2018). Estimating the transfer range of plasmids encoding antimicrobial resistance in a wastewater treatment plant microbial community. Environmental Science \& Technology Letters, 5(5), 260-265. https://doi.org/10.1021/acs.estlett.8b00105

Liao, T. L., Lin, A. C., Chen, E., Huang, T. W., Liu, Y. M., Chang, Y. H., ... Chena, Y. T. (2012). Complete genome sequence of Klebsiella oxytoca E718, a New Delhi Metallo-" ${ }^{\prime}$-Lactamase-1-producing nosocomial strain. Journal of Bacteriology, 194(19), 5454. https://doi. org/10.1128/JB.01216-12

Liu, B., Wu, H., Zhai, Y., He, Z., Sun, H., Cai, T., ... Hu, G. (2018). Prevalence and molecular characterization of oqx $A B$ in clinical Escherichia coli isolates from companion animals and humans in Henan Province, China. Antimicrobial Resistance \& Infection Control, 7, 18. https://doi. org/10.1186/s13756-018-0310-8

Liu, G., Yang, F., Li, F., Li, Z., Lang, Y., Shen, B., ... Cao, Z. (2018). Therapeutic potential of a scorpion venom-derived antimicrobial peptide and its homologs against antibiotic-resistant gram-positive bacteria. Frontiers in Microbiology, 9, 1159. https://doi.org/10.3389/ fmicb.2018.01159

Liu, L., Feng, Y., Hu, Y., Kang, M., Xie, Y., \& Zong, Z. (2018). Klebsiella grimontii, a new species acquired Carbapenem resistance. Frontiers in Microbiology, 9, 2170. https://doi.org/10.3389/fmicb.2018.02170
Liverpool School of Tropical Medicine (2019). Swab \& Send. Retrieved from https://www.lstmed.ac.uk/public-engagement/swab-send.

Lowe, C., Willey, B., O'Shaughnessy, A., Lee, W., Lum, M., Pike, K., ... Team, M. S. H. I. C. (2012). Outbreak of extended-spectrum beta-lactamase-producing Klebsiella oxytoca infections associated with contaminated handwashing sinks(1). Emerging Infectious Diseases, 18(8), 1242-1247. https://doi.org/10.3201/eid1808.111268

Martin, F. A., Posadas, D. M., Carrica, M. C., Cravero, S. L., O'Callaghan, D., \& Zorreguieta, A. (2009). Interplay between two RND systems mediating antimicrobial resistance in Brucella suis. Journal of Bacteriology, 191(8), 2530-2540. https://doi.org/10.1128/JB.01198-08

Mikheenko, A., Prjibelski, A., Saveliev, V., Antipov, D., \& Gurevich, A. (2018). Versatile genome assembly evaluation with QUAST-LG. Bioinformatics, 34(13), i142-i150. https://doi.org/10.1093/bioin formatics/bty 266

Ough, T. (2018). Is your office water bottle a health hazard? We swabbed ours to find out. The Telegraph. Retrieved from https://www.teleg raph.co.uk/health-fitness/body/office-water-bottle-health-hazar d-swabbed-find/

Pal, C., Bengtsson-Palme, J., Rensing, C., Kristiansson, E., \& Larsson, D. G. (2014). BacMet: Antibacterial biocide and metal resistance genes database. Nucleic Acids Research, 42(D1), D737-D743. https://doi. org/10.1093/nar/gkt1252

Passet, V., \& Brisse, S. (2018). Description of Klebsiella grimontii sp. nov. International Journal of Systematic and Evolutionary Microbiology, 68(1), 377-381. https://doi.org/10.1099/ijsem.0.002517

Samra, Z., Heifetz, M., Talmor, J., Bain, E., \& Bahar, J. (1998). Evaluation of use of a new chromogenic agar in detection of urinary tract pathogens. Journal of Clinical Microbiology, 36(4), 990-994. https://doi. org/10.1128/JCM.36.4.990-994.1998

Seemann, T. (2014). Prokka: Rapid prokaryotic genome annotation. Bioinformatics, 30(14), 2068-2069. https://doi.org/10.1093/bioin formatics/btu153

Tse, H., Gu, Q., Sze, K. H., Chu, I. K., Kao, R. Y., Lee, K. C., ... Yuen, K. Y. (2017). A tricyclic pyrrolobenzodiazepine produced by Klebsiella oxytoca is associated with cytotoxicity in antibiotic-associated hemorrhagic colitis. Journal of Biological Chemistry, 292(47), 19503-19520. https://doi.org/10.1074/jbc.M117.791558

Wick, R. R., Judd, L. M., Gorrie, C. L., \& Holt, K. E. (2017). Unicycler: Resolving bacterial genome assemblies from short and long sequencing reads. PLoS Computational Biology, 13(6), e1005595. https://doi. org/10.1371/journal.pcbi.1005595

Wick, R. R., Schultz, M. B., Zobel, J., \& Holt, K. E. (2015). Bandage: Interactive visualization of de novo genome assemblies. Bioinformatics, 31(20), 3350-3352. https://doi.org/10.1093/bioin formatics/btv383

Wu, M. C., Lin, T. L., Hsieh, P. F., Yang, H. C., \& Wang, J. T. (2011). Isolation of genes involved in biofilm formation of a Klebsiella pneumoniae strain causing pyogenic liver abscess. PLoS ONE, 6(8), e23500. https://doi.org/10.1371/journal.pone.0023500

Zankari, E., Hasman, H., Cosentino, S., Vestergaard, M., Rasmussen, S., Lund, O., ... Larsen, M. V. (2012). Identification of acquired antimicrobial resistance genes. Journal of Antimicrobial Chemotherapy, 67(11), 2640-2644. https://doi.org/10.1093/jac/dks261

How to cite this article: Hubbard ATM, Newire E, Botelho J, et al. Isolation of an antimicrobial-resistant, biofilm-forming, Klebsiella grimontii isolate from a reusable water bottle. MicrobiologyOpen. 2020;00:e1023. https://doi.org/10.1002/ $\underline{\text { mbo3.1023 }}$ 


\section{APPENDIX 1}

(a)

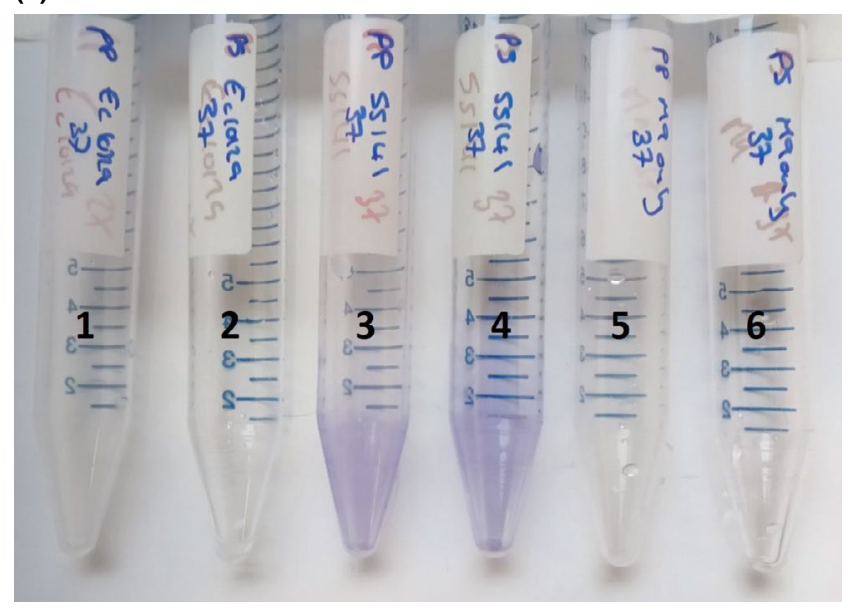

(b)

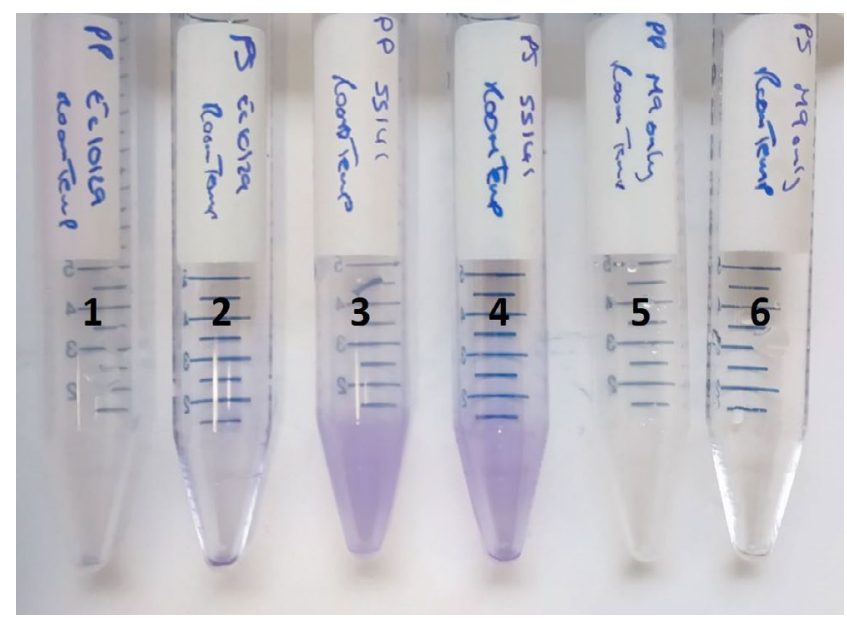

FIGURE A1 Photographs of the second biological replicate of the 15-ml tube-based biofilm formation experiment. (1) $E$. coli 10129 in polypropylene, (2) E. coli 10129 in polystyrene, (3) SS141 in polypropylene, (4) SS141 in polystyrene, (5) M9 only in polypropylene, and (6) M9 only in polystyrene incubated at (a) $37^{\circ} \mathrm{C}$ and (b) room temperature for $24 \mathrm{hr}$ (a)

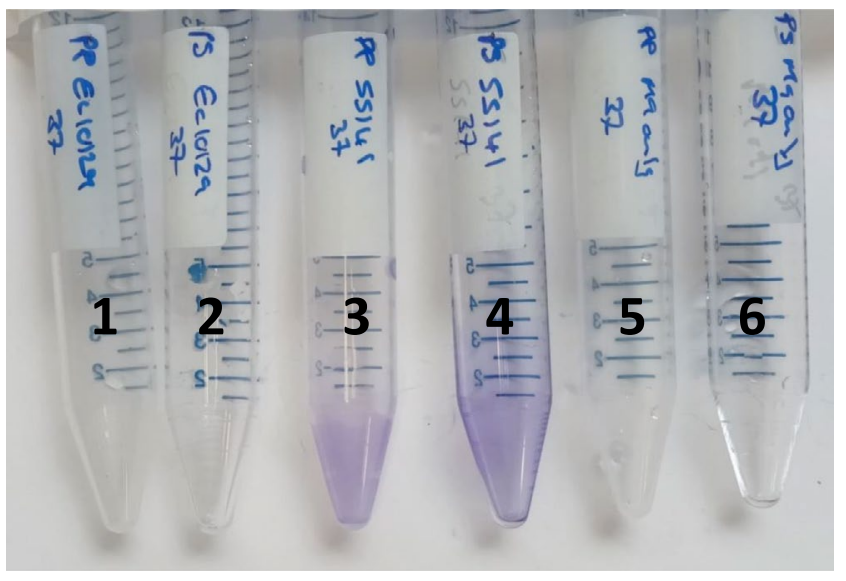

(b)

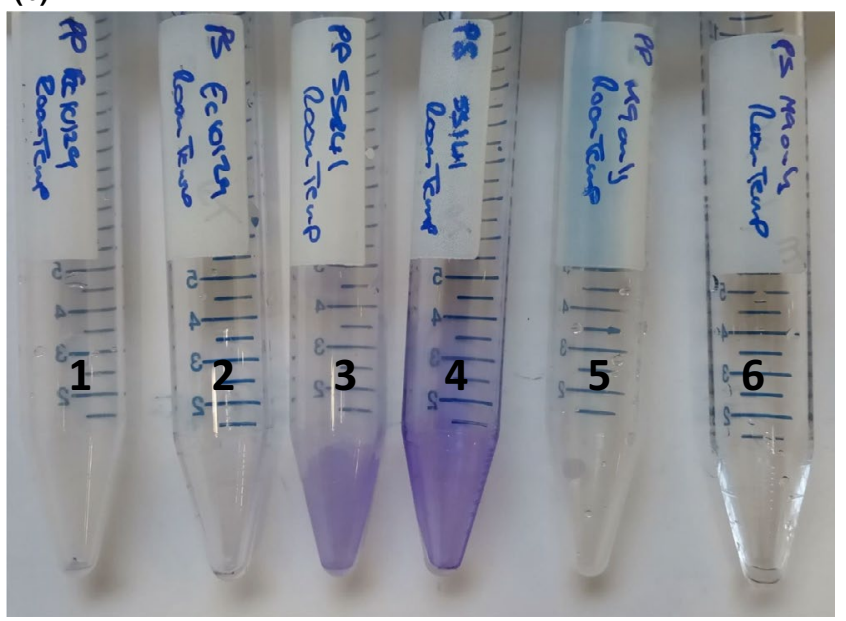

FIGURE A2 Photographs of the third biological replicate of the 15-ml tube-based biofilm formation experiment. (1) $E$. coli 10129 in polypropylene, (2) E. coli 10129 in polystyrene, (3) SS141 in polypropylene, (4) SS141 in polystyrene, (5) M9 only in polypropylene, and (6) M9 only in polystyrene incubated at (a) $37^{\circ} \mathrm{C}$ and (b) room temperature for $24 \mathrm{hr}$ 